\title{
Antiepileptic Effects of Lacosamide Loaded Polymers Implanted Subdurally in GAERS
}

\author{
Sebastien H. Bauquier, ${ }^{1}$ Jonathan L. Jiang, ${ }^{2}$ Zhilian Yue, ${ }^{3}$ Alan Lai, ${ }^{2}$ \\ Yu Chen, ${ }^{3}$ Simon E. Moulton, ${ }^{3,4}$ Karen J. McLean, ${ }^{2}$ Sara Vogrin, ${ }^{2}$ Amy J. Halliday, ${ }^{2}$ \\ Gordon Wallace, ${ }^{3}$ and Mark J. Cook ${ }^{2}$ \\ ${ }^{1}$ Faculty of Veterinary Science, University of Melbourne, 250 Princes Highway, Werribee, VIC 3030, Australia \\ ${ }^{2}$ Centre for Clinical Neurosciences and Neurological Research, St. Vincent's Hospital Melbourne, P.O. Box 2900, Fitzroy, \\ VIC 3065, Australia \\ ${ }^{3}$ Intelligent Polymer Research Institute and ARC Centre of Excellence for Electromaterials Science, AIIM Facility, \\ University of Wollongong, Innovation Campus, Wollongong, NSW 2522, Australia \\ ${ }^{4}$ Faculty of Science, Engineering and Technology, Swinburne University of Technology, Hawthorn, VIC 3122, Australia
}

Correspondence should be addressed to Sebastien H. Bauquier; bauquier@unimelb.edu.au and Simon E. Moulton; smoulton@swin.edu.au

Received 30 September 2015; Revised 18 December 2015; Accepted 22 December 2015

Academic Editor: Alenka Vesel

Copyright (C) 2016 Sebastien H. Bauquier et al. This is an open access article distributed under the Creative Commons Attribution License, which permits unrestricted use, distribution, and reproduction in any medium, provided the original work is properly cited.

\begin{abstract}
The current experiment investigated the ability of coaxial electrospun poly(D,L-lactide-co-glycolide) (PLGA) biodegradable polymer implants loaded with the antiepileptic drugs (AED) lacosamide to reduce seizures following implantation above the motor cortex in the Genetic Absence Epilepsy Rat from Strasbourg (GAERS). In this prospective, randomized, masked experiments, GAERS underwent surgery for implantation of skull electrodes $(n=6)$, skull electrodes and blank polymers $(n=6)$, or skull electrodes and lacosamide loaded polymers $(n=6)$. Thirty-minute electroencephalogram (EEG) recordings were started at day 7 after surgery and continued for eight weeks. The number of SWDs and mean duration of one SWD were compared week-byweek between the three groups. There was no difference in the number of SWDs between any of the groups. However, the mean duration of one SWD was significantly lower in the lacosamide polymer group for up to 7 weeks when compared to the control group $(0.004<p<0.038)$. The mean duration of one seizure was also lower at weeks $3,5,6$, and 7 when compared to the blank polymer group ( $p=0.016,0.037,0.025$, and 0.025 , resp.). We have demonstrated that AED loaded PLGA polymer sheets implanted on the surface of the cortex could affect seizure activity in GAERS for a sustained period.
\end{abstract}

\section{Introduction}

Epilepsy is a chronic neurological condition characterized by recurrent seizures. The incidence of epilepsy in most developed countries is between 50 and 100 cases per 100,000 population per year although it is estimated that up to $5 \%$ of a population will experience nonfebrile seizures at some point in life $[1,2]$. Individuals with medically untreatable epilepsy often have impaired ability to work or function socially (e.g., inability to drive, difficulty at attending school, losing jobs and friends, and anxiety regarding the possibility of having seizure in potentially hazardous conditions) [3]. Treatment with conventional antiepileptic drugs (AEDs, e.g., phenytoin and lacosamide administered orally) results in only $33 \%$ of the patients having no seizure recurrence $[1,2]$. Alternatively, neurostimulation based therapy has also been shown to reduce seizure activity but has typical reductions of seizure frequency of approximately $40 \%$ acutely and 50-69\% after several years [4]. Surgical resection of the seizure focus can be performed in the case of focal seizures; however, this procedure can only be applied on selected patients depending on the localization of the epileptic foci [5]. Indeed, the success 
of inducing long-lasting seizure remission from epilepsy surgery ranges from a low of $25 \%$ for patients exhibiting extrahippocampal seizure origin to $70 \%$ in appropriately selected candidates [5].

The mechanisms by which resistance to AEDs treatment develops are not fully understood; some evidence suggests that this may be due to a lack of effective penetration into the brain parenchyma; however, the drug side effects prevent large increase in the posology [6]. Alternative therapies aiming at improving the availability of AEDs such as the intracranial implantation of polymer-based drug delivery systems are being investigated [7, 8]. This targeted drug delivery approach has shown some success in the treatment of animal models of several neurological disorders such as Parkinson's disease, Huntington's disease, and Alzheimer's disease [9]. Also, Halliday et al. used Levetiracetam loaded biodegradable polymer implants in the tetanus toxin model of temporal lobe epilepsy in rats; the results of this study indicated that drug-eluting polymer implants represent a promising evolving treatment option for intractable epilepsy; however, important limitations of the study were that the effects could only be seen for a week and only a single group of control animals were investigated. These animals received an injection of tetanus toxin and a sham craniotomy, without the implantation of a polymer sheet [8].

Poly(D,L-lactide-co-glycolide) (PLGA) is the most commonly used biodegradable polymer as it is highly biocompatible and easily engineered and has been approved for drug delivery purposes by the United States Food and Drug Administration [10]. It has been used in numerous applications including bone and skin tissue engineering, ocular treatment, vaccine, cancer therapy, and nerve regeneration [11-15]. PLGA polymers have also been successfully used for intracranial drug delivery in animal models of neurological disorders, showing no evidence of toxic injury or immunemediated inflammation when implanted subdurally above the motor cortex in rats [10].

Genetic Absent Epilepsy Rat from Strasbourg (GAERS) is a strain of rats where $100 \%$ of the animals present with recurrent generalized nonconvulsive seizures [16]. This animal model has become the gold standard to study the mechanisms underlying absence epilepsy [16]. In the present investigation, PLGA sheets loaded with the commonly used AED lacosamide were developed and their ability to decrease seizure activity was investigated in the GAERS. Lacosamide stabilizes neuronal membranes through enhancing slow inactivation of voltage-gated sodium channel and is effective in different rodent seizure models including generalized seizure [17].

The aim of the study is to investigate the effect of subdural implantation of biodegradable polymers (PLGA) loaded with lacosamide on seizure activity in an animal model of epilepsy (GAERS); the hypothesis is that subdural implantation of lacosamide loaded PLGA polymers can decrease seizure activity of the GAERS. Our results demonstrated that focal delivery of lacosamide can achieve partial sustained antiepileptic effect in an animal model of generalized epilepsy.

\section{Materials and Methods}

The study was designed as a randomized controlled masked experiment. The experiment was approved by St. Vincent's Hospital (Melbourne) Animal Ethics Committee and conducted in accordance with the Australian Code of Practice for the Care and Use of Animals for Scientific Purposes (2004).

2.1. PLGA Polymer Mat Production. Lacosamide-laden polymer mats were produced using a coaxial electrospinning method [18], with the core composed of lacosamide and $75: 25$ PLGA (lactide/glycolide $=75: 25$ ) and the shell composed of $85: 15$ PLGA (lactide/glycolide $=85: 15$ ). The core solution was prepared at 17\% w/v $75: 25$ PLGA in dimethylformamide (DMF), to which lacosamide was added to give a range of final concentration of $2.5,12.5$, or $20 \% \mathrm{w} / \mathrm{w}$ relative to the polymer. The shell solution was prepared at 20\% w/v $85: 15$ PLGA in a binary solvent system comprising dichloromethane and DMF (dichloromethane/DMF $=7 / 3$ ). Coaxial electrospinning was conducted using a nanoelectrospinning system (NANON-01A, MECC Co. Ltd.) at an applied DC voltage of $23 \mathrm{kV}$. A coaxial spinneret with a diameter of $0.2 \mathrm{~mm}$ for the core and $0.8 \mathrm{~mm}$ for the sheath nozzles was connected to the core and shell solutions and a feed rate of $5 \mu \mathrm{L} \mathrm{min}^{-1}$ and $20 \mu \mathrm{L} \mathrm{min}^{-1}$ for the core and shell solutions, respectively. Fiber mats were collected on a grounded plate collector that was set $15 \mathrm{~cm}$ away from the spinneret tip. The drug-free, coaxially spun polymer mats were also prepared using the above procedure.

Depending on lacosamide loading in the core, the asprepared, coaxially electrospun polymer mats were denoted as PLGA-2.5\%, PLGA-12.5\%, and PLGA-20\%. The drug-free mats are denoted as blank polymers. All the samples were finally dried in a vacuum oven at $40^{\circ} \mathrm{C}$ for $72 \mathrm{~h}$ to remove residual solvent and were stored at $-20^{\circ} \mathrm{C}$ prior to subsequent physiochemical characterization and animal studies.

2.2. Morphology Study. The morphology of the electrospun mats (with and without drug) was examined using a field emission scanning electron microscope (FESEM, JEOL JSM7500FA). The samples were sputter-coated with gold prior to FESEM to avoid sample charging.

2.3. Determination of Drug Loading in Polymer Mats. An extraction method was used to determine the drug loading in the electrospun mats. Briefly, each sample $(1 \mathrm{~cm} \times 1 \mathrm{~cm})$ was weighed and placed into $1 \mathrm{~mL}$ methanol for 12 hours after which the methanol was removed and replenished with $1 \mathrm{~mL}$ of fresh methanol. This extraction procedure was repeated four times with each methanol sample allowed to evaporate to leave residual drug behind which was reconstituted in methanol $(1 \mathrm{~mL})$, diluted 20 times with the HPLC mobile phase (see below), and filtered through a $0.2 \mu \mathrm{m}$ syringe filter. The fourth reconstituted sample showed no presence of drug indicating that the entire drug had been extracted from the electrojetted sample. 
2.4. In Vitro Drug Release. The lacosamide loaded polymers $(1 \mathrm{~cm} \times 1 \mathrm{~cm})$ were suspended in $1 \mathrm{~mL}$ of aCSF, and the release experiment was conducted at $37^{\circ} \mathrm{C}$ in a shaker water bath (Julabo Pty. Ltd.). The aCSF contained $\mathrm{NaCl}(0.866 \% \mathrm{w} / \mathrm{v})$, $\mathrm{KCl}(0.224 \% \mathrm{w} / \mathrm{v}), \mathrm{CaCl}_{2}-2 \mathrm{H}_{2} \mathrm{O} ;(0.0206 \% \mathrm{w} / \mathrm{v})$, and $\mathrm{MgCl}_{2}-$ $6 \mathrm{H}_{2} \mathrm{O}(0.0164 \% \mathrm{w} / \mathrm{v})$ in $1 \mathrm{mM}$ phosphate buffer $(\mathrm{pH} 7.4)$ [8]. For each sample, the release medium solution was collected and replenished with fresh aCSF at various time points and stored at $-20^{\circ} \mathrm{C}$ prior to HPLC analysis being undertaken. The eluted samples were analyzed by HPLC using a modified method on an Agilent 1260 Infinity HPLC system [19]. An Atlantis ${ }^{\circledR}$ T3 C18 column $(250 \mathrm{~mm} \times 4.6 \mathrm{~mm}, 5 \mu \mathrm{m})$ was employed as the analytic column and set at $40^{\circ} \mathrm{C}$. The mobile phase was composed of water, acetonitrile, and methanol $(65: 26.2: 8.8, \mathrm{v} / \mathrm{v} / \mathrm{v})$, and the injection volume was $10 \mu \mathrm{L}$ with the flow rate of $0.8 \mathrm{~mL} / \mathrm{min}$. The eluting lacosamide was detected using a UV-vis detector set at a wavelength of $230 \mathrm{~nm}$ ( $\lambda_{\max }$ of lacosamide). To convert the UV-vis absorbance to drug concentration a standard curve was established by plotting in triplicate the UV-vis peak areas against respective concentrations of standard solutions (10, $20,50,100,200$, and $500 \mu \mathrm{M}$ lacosamide).

2.5. Animals. Adult female GAERS were obtained from the University of Melbourne (Parkville, Victoria, Australia) and housed individually in inverted 12-hour light/dark cycles (the light was turned off between 6 am and $6 \mathrm{pm}$ ) with ad libitum access to food and water. Six-month-old rats were randomly allocated to a control group (no implant; $n=$ 6 ), blank polymer group (bilateral implantation of blank PLGA polymers not containing lacosamide; $n=6$ ), or treatment group (bilateral implantation of lacosamide loaded PLGA polymers; $n=6$ ). The randomization was performed using the random function in Microsoft Excel 2007. The group attribution list was kept concealed from the researchers performing the EEG analysis.

2.5.1. Implantation Surgery. Immediately prior to surgery, rats were weighted and anaesthetized using a balanced anesthesia protocol including an intraperitoneal injection of ketamine $(75 \mathrm{mg} / \mathrm{kg})$ and xylazine $(10 \mathrm{mg} / \mathrm{kg})$. Following anesthesia induction rats were placed in a stereotaxic apparatus, given isoflurane ( 0.5 to $1 \%$ in oxygen, $1 \mathrm{~L} / \mathrm{min}$ ) via a nose-cone as needed, and given subcutaneous (SQ) carprofen $(5 \mathrm{mg} / \mathrm{kg})$ for pain relief and $0.9 \%$ sodium chloride $(2 \mathrm{~mL})$ for cardiovascular support.

Rats from the control group underwent surgery for EEG recording electrode implantation whereas surgeries for the rats from the blank polymer group, the treatment group, and the silicone group also included bilateral craniotomies for subdural placement of two identical implants. Over the scalp of all rats, the hair was clipped and the skin was aseptically prepared. A single incision was made down the midline, the skull cleared of tissue, and the exposed bone dried with $3 \%$ hydrogen peroxide. Five extradural electrodes, consisting of small jeweller's screws, were implanted caudal to the intended polymer implantation sites through burr holes. Four were implanted cranially to the interaural line (two on each side of the sagittal suture) and one was implanted caudally to the interaural line on the right side of the sagittal suture (Figures 1(a) and 1(b)). The electrodes were then connected to an adaptor and secured with dental cement.

Implants measuring $3 \mathrm{~mm}$ by $4 \mathrm{~mm}$ were cut from the polymer sheets described above. Placements of the implants were performed after $5 \mathrm{~mm}$ by $4 \mathrm{~mm}$ craniotomies were created bilaterally at the level of the coronal suture (over the motor cortices) and after excising the dura to expose the brain surface (Figures 1(a) and 1(b)). Following implant placements, the skull removed from craniotomy sites was replaced. The craniotomy sites were then sealed with an alginate-based hydrogel, the entire surgical site was covered with dental cement, and the skin was sutured leaving exposed only part of the dental cement. The animals were placed on heat pads for recovery. Postoperative treatment included SQ buprenorphine every twelve hours $(0.03 \mathrm{mg} / \mathrm{kg}$, twice a day), saline ( $2 \mathrm{~mL}$, once a day), and carprophen SID (5 mg/kg, once a day) for up to 3 days.

2.5.2. Electroencephalograph Recording and Analysis. At day 7 or 8 after surgery and at least 3 days per week for the following 7 weeks, rats were monitored for one hour (half an hour anesthesia recovery/acclimation time and half an hour recording time) (Figure 1(c)). At each monitoring session, rats were briefly anaesthetized in an induction cage with isoflurane ( $4 \%$ in oxygen, $2 \mathrm{~L} / \mathrm{min}$, for 2 to 3 minutes), and shielded cables were used to connect the recording electrodes to the EEG acquisition system, which consisted of TDT processors and high impedance head stages driven by custom-designed software (Tucker Davis Technologies, USA). The rats were allowed to recover from the anesthetic before recordings began. The EEGs were visualized using a custom-designed MATLAB program (The MathWorks, Inc., USA). During EEG recording, if the rats were perceived as being asleep, and after confirmation of no seizure activity on the EEG, a noise stimulus between 94 and 98 decibels was applied. At the end of a recording session, the rats were again briefly anaesthetized with isoflurane ( $4 \%$ in oxygen, $2 \mathrm{~L} / \mathrm{min}$ ) to be disconnected from the shielded cable. The researcher performing EEG analysis was masked to the treatment and used a GAERS specific automated spike-and-wave discharges (SWDs) detection algorithm [20].

2.6. Primary Outcome: Epileptic Activity. For each rat, the median value and interquartile range for number of SWDs, duration of one SWD, and cumulative duration of SWDs over 30 min were calculated for eight recording blocks, each block representing 1 week of recording. The first block only included one recording at day 6 or 7 after surgery (total of 30 minutes of EEG recording) whereas the following 7 blocks included 3 recordings per week (total of 90 minutes of EEG recording per week) (Figure 1(c)).

2.7. Secondary Outcome: Postoperative Health Monitoring. For a minimum of three days after surgery and until full recovery from the surgery, the rats were monitored for weight loss once a day and for mobility and grooming twice 


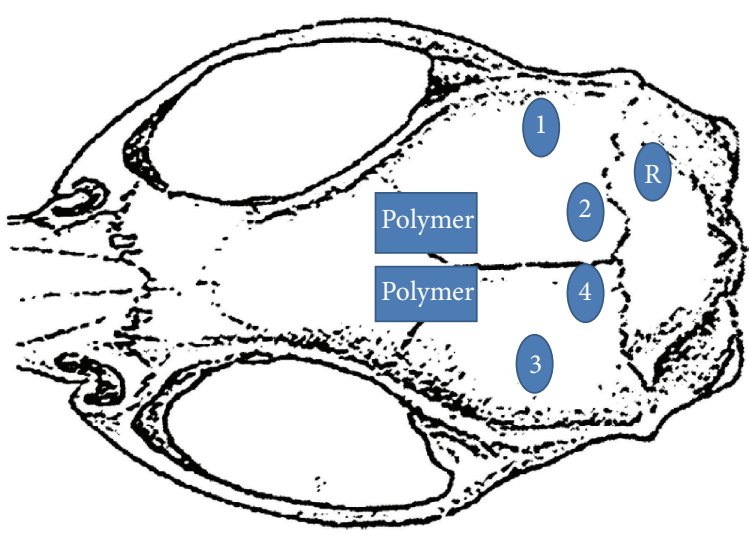

(a)

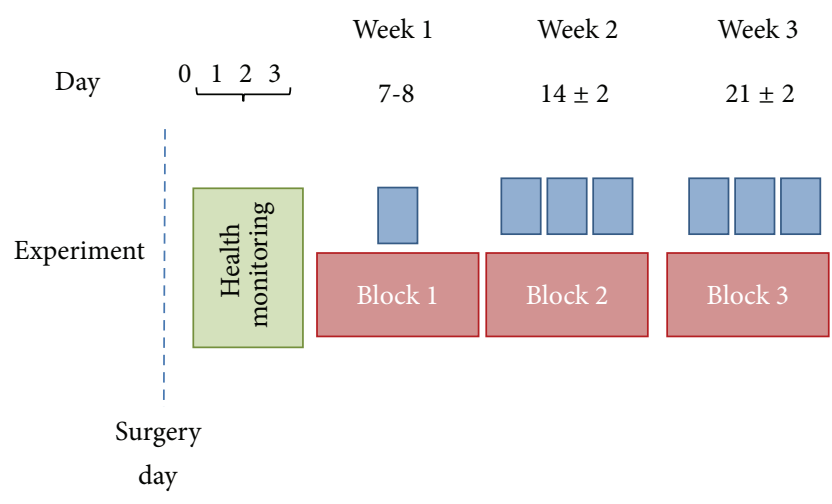

Each square represents 30 min EEG recording

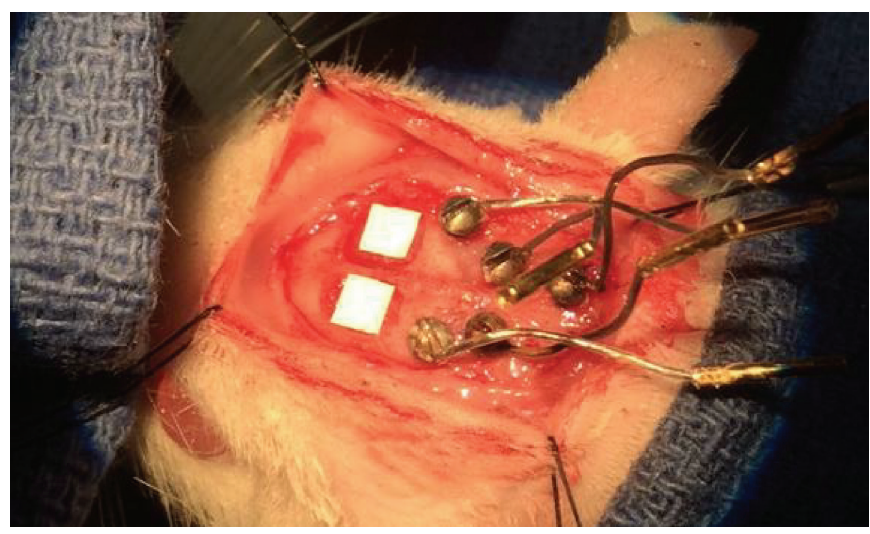

(b)

Week $4 \quad$ Week 5

Week 6

Week 7

Week 8

$28 \pm 2 \quad 35 \pm 2$

$42 \pm 2$

$49 \pm 2$

$56 \pm 2$
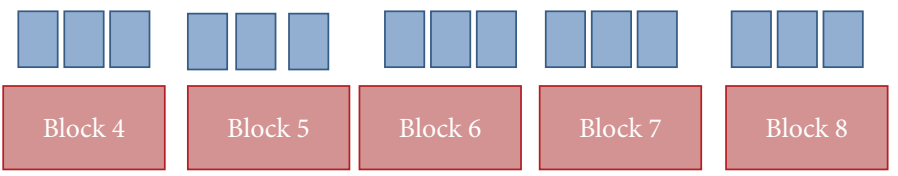

(c)

FIGURE 1: Schematic diagram illustrating the positioning of epidural recording screw electrodes (1-4), reference electrode (R) and polymer (a); picture of the actual surgery (b); timeline of the study design: for a minimum of three days after surgery the rats were monitored for weight loss once a day and for mobility and grooming twice a day. Thirty-minute electroencephalogram recording began at day 7 or 8 after surgery and for the following 7 weeks on 3 days per week. For each rat, the mean desired values (number of SWDs, duration of one SWD, or cumulative duration of SWDs over $30 \mathrm{~min}$ ) were calculated for eight recording blocks, each block being 1 week of duration.

a day. Those observations were given scores (Table 1). For each day a debilitation score was calculated by cumulating the highest grooming and mobility scores with the weight loss score (Table 1). A debilitation score of 0,1 to 3,4 to 6 , and 7 to 9 meant that the rat health was not affected, mildly affected, moderately affected, and severely affected by the surgery/polymer implantation, respectively. The highest debilitation score of each rat over the postoperative period is reported.

2.8. Statistical Analysis. Data analysis was performed using a commercially available software (IBM SPSS Statistic 22; Stata 13.0; StataCorp, 2013). The number of SWDs, mean duration of one SWD, and cumulative duration of SWDs were compared between the three groups using individual Kruskal-Wallis tests for each block of the study. When significant values were found, post hoc pairwise comparisons were conducted for this block, comparing the control versus lacosamide polymer and blank polymer versus lacosamide polymer conditions using the Mann-Whitney $U$ test. The debilitation scores of group lacosamide were compared to group control and group blank polymer using a 2-tailed Mann-Whitney exact test. Significance level was set at 5\%.

\section{Results}

The FESEM images obtained from the PLGA mats without lacosamide (Figure 2(a)) show a smooth and regular morphology characteristic of PLGA electrospun fibers. However, when lacosamide was incorporated into the PLGA the fibers within the mats demonstrated a mixture of porous and nonporous morphology when examined under FESEM (Figure 2(b)). Measured by visual inspection of the FESEM images, the fiber diameter ranged between $2 \mu \mathrm{m}$ and $5 \mu \mathrm{m}$. These observations of the PLGA-12.5\% samples shown in Figure 2(c) were also observed in the other PLGA-2.5\% and PLGA-20.0\% samples.

The in vitro release results for the PLGA-lacosamide mats are presented in Figure 2(c). The polymer containing 20\% lacosamide showed a cumulative drug release above $40 \%$ after only 7 days. The polymers containing 2.5 and $12.5 \%$ lacosamide both had a reduction in release after around 42 


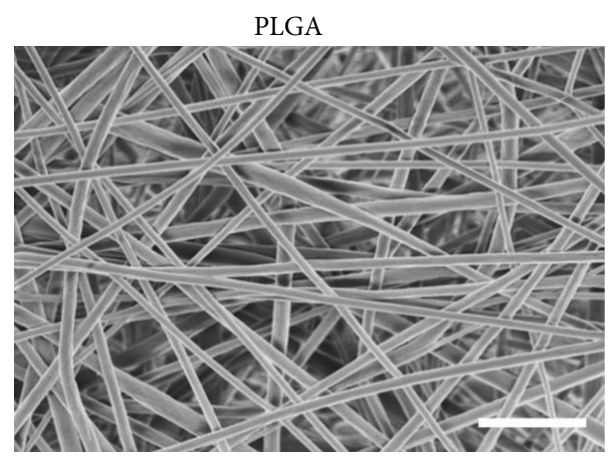

(a)

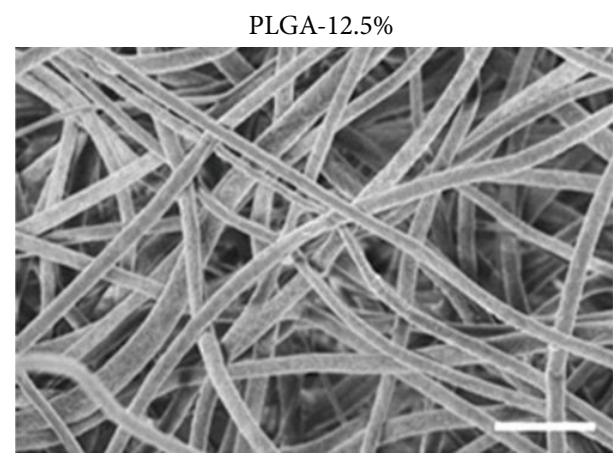

(b)

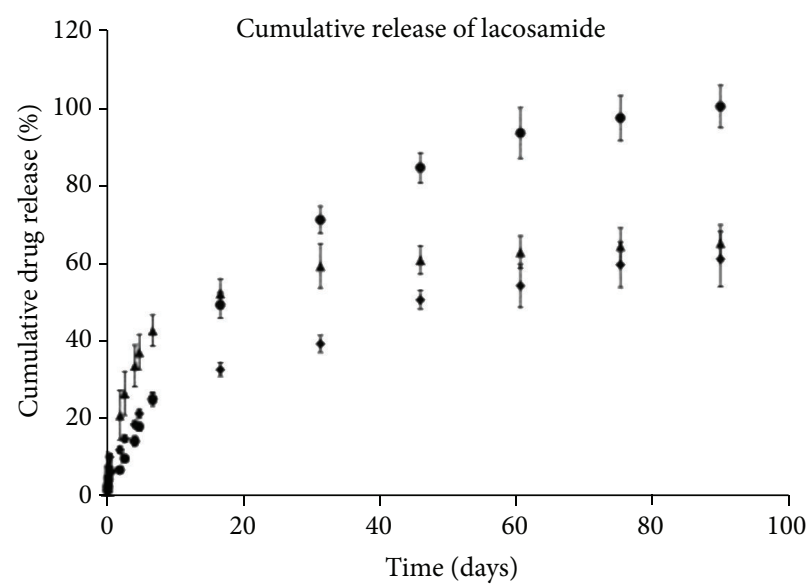

- PLGA-2.5\%

- PLGA- $12.5 \%$

- PLGA-20\%

(c)

FIGURE 2: Scanning electron micrographs of coaxially electrospun polymer mats of PLGA Blank (a) and PLGA-12.5\% lacosamide (b) (scale bar represents $10 \mu \mathrm{m}$ ). In vitro release data of lacosamide polymers with final core lacosamide concentration of $2.5,12.5$, and $20 \% \mathrm{w} / \mathrm{w}$ relative to the polymer $(\mathrm{c})$. Bars are representing standard deviation.

days (6 weeks); however, the total drug release was higher for $12.5 \%$ lacosamide polymer. In consequence, the polymer containing $12.5 \%$ lacosamide was chosen to be implanted for the in vivo experiment.

The results for the postoperative debilitation score are presented in Figure 3. One rat from the blank polymer group and 3 rats from the lacosamide had a debilitation score which were classified as moderate. One rat from the blank polymer group and 3 rats from the lacosamide group had a debilitation score which were classified as severe. The debilitation scores were significantly increased for the lacosamide polymer group when compared to the control and blank polymer groups ( $p=0.002$ and $p=0.041$, resp.).

The results of the measurements performed to evaluate the epileptic activity for the groups control, blank polymer, and lacosamide polymer are presented in Figure 4. The difference in the number of SWDs between the groups was not statistically significant. However, the mean duration of one SWD was significantly lower in the lacosamide polymer group for up to 7 weeks when compared to the control group

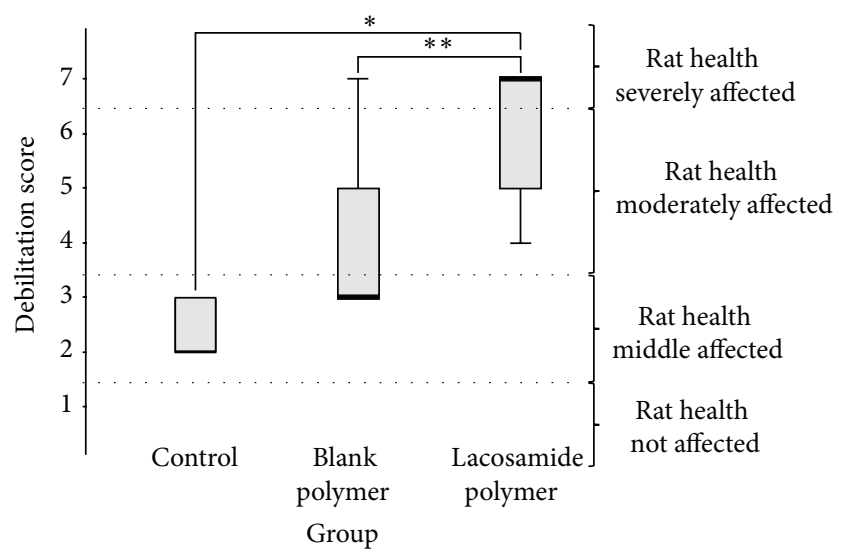

FIGURE 3: Postoperative debilitation scores obtained for groups control $(n=6)$, blank polymer $(n=6)$, and lacosamide polymer $(n=6)$. Rats from the lacosamide polymer group had debilitation scores that were higher when compared to the 2 other groups $\left({ }^{*} P=\right.$ $\left.0.002 ;{ }^{* *} p=0.041\right)$. The box indicates the interquartile range (25th to 75 th percentile), and the whiskers indicate the range. 


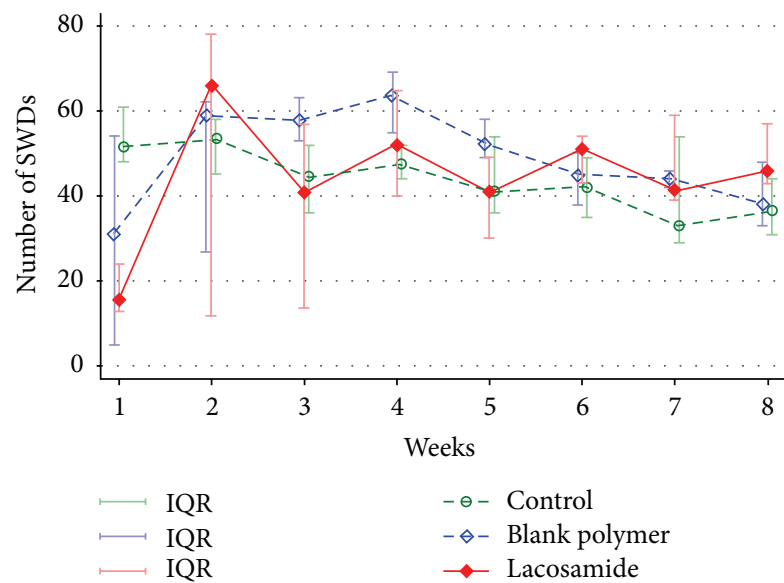

(a) Number of SWDs

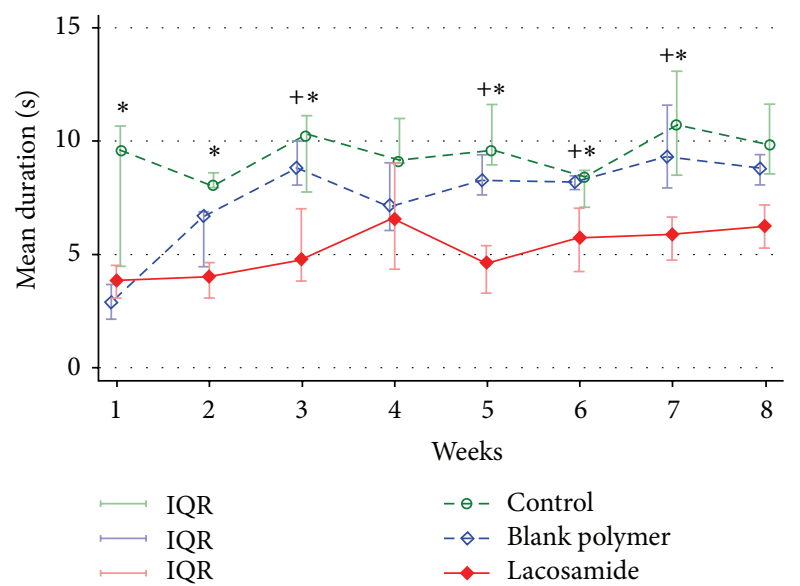

(b) Mean duration of one SWD

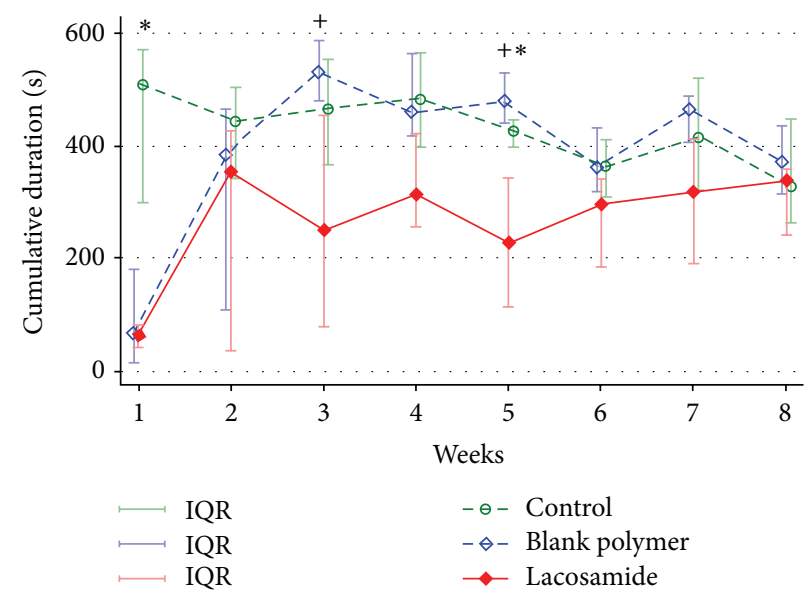

(c) Cumulative duration of SWDs

Figure 4: Comparison of the measurements obtained to evaluate the epileptic activity of groups control $(n=6)$, blank polymer $(n=6)$, and lacosamide polymer $(n=6)$. SWD: spike-and-wave discharge; IQR: interquartile range; results are reported as median. * and + represent the time points at which the results from the lacosamide polymer group were significantly different to the control and blank polymer groups, respectively $(p<0.05)$.

( $p=0.037,0.004,0.01,0.025,0.037$, and 0.016 for weeks 1 , $2,3,4,6$, and 7 , resp.). The mean duration of one seizure was also lower at weeks $3,5,6$, and 7 when compared to the blank polymer group $(p=0.016,0.037,0.025$, and 0.025 , resp.). The cumulative duration of SWDs of the lacosamide group was significantly lower when compared to the control group at weeks 1 and 5 ( $p=0.010$ and $p=0.055$, resp.) and when compared to the blank polymer group at weeks 3 and 5 ( $p=0.010$ and $p=0.055$, resp.). Examples of EEG recordings are presented in Figure 5.

\section{Discussion}

Drug release from electrospun polymeric structures typically follows zero-order kinetics [18]. Zero-order kinetics implies a homogeneous drug distribution and a release profile governed by the wetting properties of the material and encapsulation of hydrophilic and neutrally charged drugs of low molecular weight can be problematic for these types of structures [21-24]. The interaction of these types of drug molecules with the polymer is usually very poor and their rate of diffusion is often faster than the rate of polymer erosion. This fast rate of diffusion has detrimental effects on drug release from electrospun polymer structures.

Several factors are responsible for the variations in the release profiles of AEDs, namely, the solubility of the drug in the mat, the morphology of the fibers within the mat, and the distribution of the drug throughout the fibers (i.e., the degree of drug encapsulation within the core of the coaxial spun mats). The solubility of lacosamide ( $465 \mathrm{mg} / \mathrm{L})$ contributes to the relatively fast elution of that drug from the polymer. Also, the morphology of the fibers within the electrospun mats shows a porous nature for the PLGA-lacosamide structures which increases the rate at which the release media (aCSF) can infuse into the internal region of the fibers and promote the elution of the drug.

The initial rapid drug release observed in the in vitro experiment for the polymer mats tested with a final core lacosamide concentration of $12.5 \%$ (Figure 2(c)) coincided 


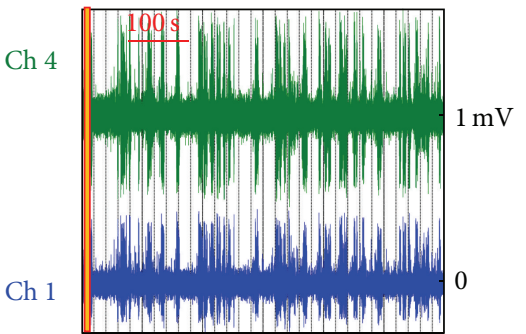

(a) Rat 9 week 5_Control

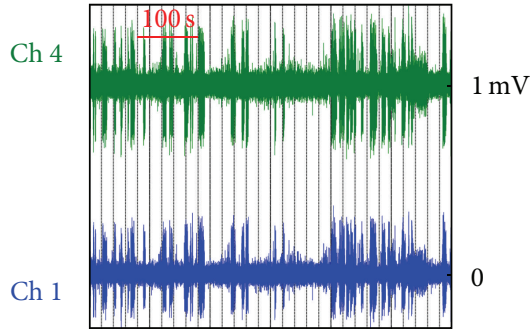

(b) Rat 5 week 5_Blank

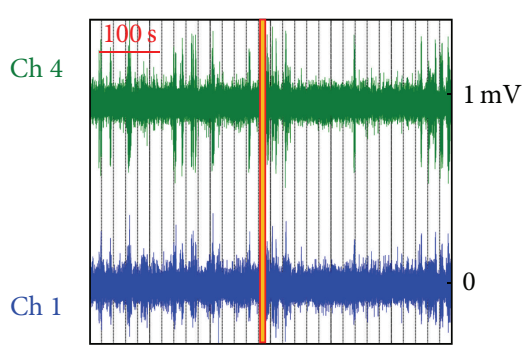

(c) Rat 11 week 5_Lacosamide

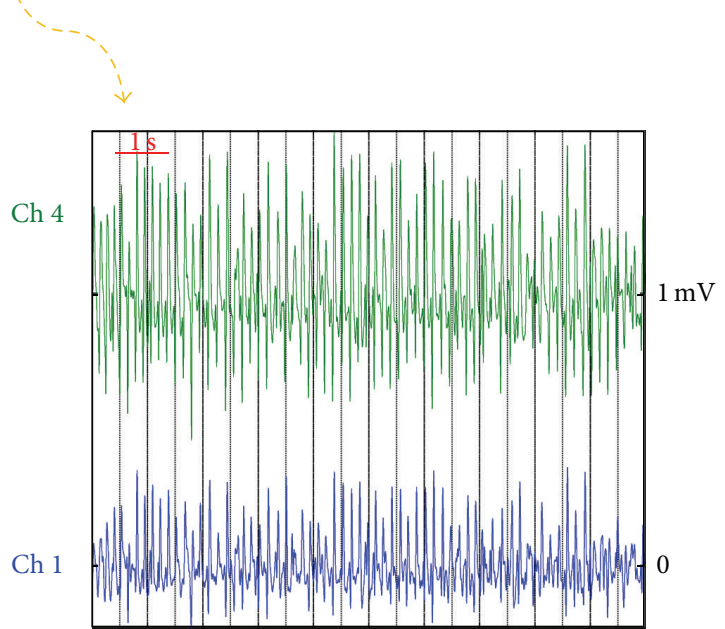

(d) Rat 9 week 5_SWD

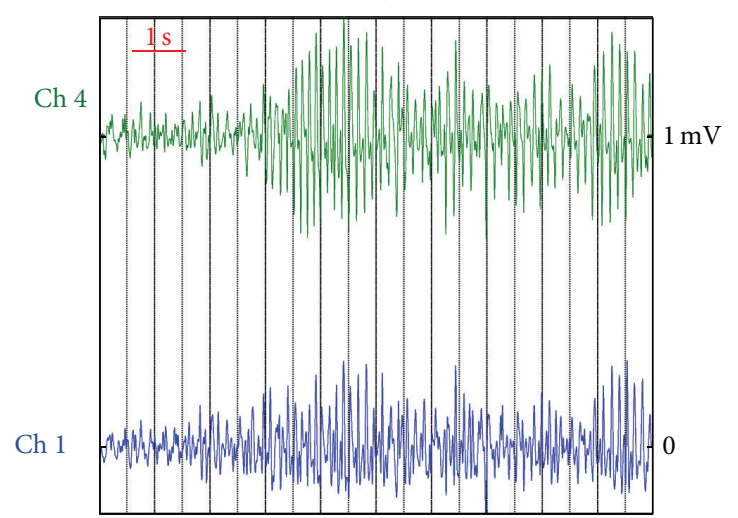

(e) Rat 11 week 5_SWD

FiguRE 5: Example of EEG recording from 3 rats belonging to group control (a), blank polymer (b), and lacosamide polymer (c). Figures (d) and (e) are amplifications of spike-and-wave discharges observed during the recording shown and Figures (a) and (c), respectively.

with the postsurgical debilitation seen in most animals of the lacosamide polymer group. It is unlikely that the surgery alone was responsible for these adverse events as this debilitation was not observed to such extent in the blank polymer group. Debilitated rats were treated with fluid therapy and analgesic administration and they all recovered well. Looking at the results retrospectively, one could argue that implanting the PLGA-2.5\% may have been a better choice as the initial release of drug is not as abrupt and the constant lacosamide release seems to last longer. Indeed the lack of effect of lacosamide after 7 weeks correlates with the almost absence of release of the lacosamide from the polymer. Variations in the release profiles observed in Figure 2(a) from the samples prepared with varying amounts of lacosamide indicate that the interaction of the drug with the polymer (and hence its propensity to be released from the structures) is influenced by the drug loading. It has previously been shown that the amount of drug loaded into electrospun fibers and drugpolymer-electrospinning solvent interactions has an effect on the release profiles [25-28].

Biodegradable PLGA polymer sheets containing a large amount of lacosamide were implanted above the motor cortices of GAERS. In the GAERS model of epilepsy, the rats present recurrent generalized nonconvulsive seizures characterized by bilateral and synchronous SWD accompanied with behavioral arrest, staring, and sometimes twitching of the vibrissae [16]. Furthermore, the GAERS were at least
6 months old, time at which $100 \%$ of the GAERS should present SWDs and at which the numbers of SWDs are at their maximum [16]. Although depth EEG recordings and lesion experiments show that SWDs in GAERS depend on cortical and thalamic structures with a possible rhythmic triggering by the lateral thalamus, more recent studies indicate a seizure initiation site within the perioral region of the somatosensory cortex (S1po) as well as the somatosensory cortex forelimb region (S1FL) [16, 29, 30]. Neurophysiological, behavioral, pharmacological, and genetic studies have demonstrated that spontaneous SWDs in GAERS fulfill all the requirements for an experimental model of absence epilepsy [16]. Although twenty-minute recordings were used by the original paper describing GAERS EEGs, to try to improve the performance of the EEG analysis 30 min recordings were used during the present experiment [16].

Although spontaneous SWDs start and end abruptly on a normal background EEG and are quite easy to isolate, the EEG patterns seen during sleep make it more difficult to differentiate start and stop of SWD [20]. In previous experiments, rats were stimulated when seen sleeping to improve seizure detection [20]. This intervention was reduced during the present experiment by inverting the light cycle of the rats. The EEG recordings of those diurnal animals were then recorded during the time of maximal activity reducing sleep time EEG interferences. SWDs usually occur at a mean frequency of 1.5 per min when the animals are in a state of 
TABLE 1: Postoperative health monitoring chart. For a minimum of three days after surgery and until full recovery from the surgery, the rats were monitored for weight loss once a day and for mobility and grooming twice a day. For each day a debilitation score was calculated by cumulating the highest grooming and mobility scores with the weight loss score. A debilitation score of 0,1 to 3,4 to 6 , and 7 to 9 meant that the rat health was not affected, middle affected, moderately affected, and severely affected by the surgery/polymer implantation, respectively.

\begin{tabular}{lc}
\hline Postoperative health monitoring chart & Score \\
\hline Percentage of weight loss & 0 \\
No weight loss & 1 \\
Less than 10\% weight loss & 2 \\
10\% to less than 20\% weight loss & 3 \\
20\% or more weight loss & \\
Mobility score & 0 \\
Rat moving normally & 1 \\
Rat ataxic moving at a normal speed & 2 \\
Rat ataxic and moving slowly & 3 \\
Rat recumbent & \\
Grooming score & 0 \\
No decrease in grooming activity & 1 \\
Middle decrease in grooming activity & 2 \\
Moderate decrease in grooming activity & 3 \\
No grooming activity & $0-9$ \\
\hline
\end{tabular}

quiet wakefulness and have duration ranging from 0.5 to $75 \mathrm{~s}$ [16].

Isoflurane was used for a short period to connect and disconnect the recording apparatus before and after each recording. The authors found in previous experiments that during that setup without the use of anaesthesia some rats were showing stress behaviour (like crying) and in consequence the authors opted for a short general anaesthesia/sedation to improve animal welfare. Isoflurane was chosen for its low blood:gas solubility (1.4) allowing quick elimination. Indeed, rats recovered quickly from those short anaesthesia episodes and, although it cannot be excluded, it is unlikely that after 30 minutes isoflurane could still be interfering with their epileptic activity [31].

Implantation of the lacosamide sheets led to shorter seizures for up to seven weeks after implantation compared to rats that did not receive the implant.

During the first 2 weeks of the experiment, the blank polymer group also demonstrated decrease duration of SWDs when compared to the control group and to better understand the effect seen, silicon sheets were implanted in four older GAERS from the same colony (unpublished data). Although the SWD activity in GAERS is age dependent, preventing statistical comparison with the present experiment, the postsurgical transient decrease in SWD's duration was observed again. We could assume that the PLGA sheets were not themselves responsible for the decreased duration of SWDs seen in the control blank polymer group but could be attributed to brain injuries resulting from the surgery. This assumption is in agreement with previous publication reporting that PLGAbased implants are very well tolerated by the brain in animal models of other neurological disorders [32-37].

Only one EEG recording per rat was performed during the first week at day 6 or 7 while there were three recordings per week performed during the following seven weeks. This study design allowed time for good surgical wound healing before some traction could be applied to the electrodes "adaptor."

The lacosamide was chosen for its lipophilic properties allowing easy drug loading within the polymer and its proven efficacy in treating absence epilepsy [38]. Knowing that the polymer mats were implanted over the motor cortex and that previous investigations have shown that substances released from intraparenchymally implanted polymers are able to penetrate around $3 \mathrm{~mm}$, it is possible that the lacosamide released from the sheets in our study may not have reached the seizure triggering focus in high enough concentration to stop seizure from happening [39-42]. However, lacosamide reduces the ability of epileptic neurons to endure extended firing burst by enhancing slow inactivation of voltage-gated sodium channel [43]. This would explain the unchanged number of SWDs but significant decrease in SWDs duration. The total duration of SWDs during the recording of SWD reflects the arithmetic product of the number of seizures and the mean duration of SWDs. Another AED alternative could have been the use of valproic acid which is one of the drugs of choice for the treatment of absence seizure. Its use could have provided additional support for the validity of the novel delivery method. Lastly, now that it has been shown that lacosamide loaded PLGA polymer sheets implanted on the surface of the cortex could affect the duration of the individual SWDs in GAERS, performing dose-response experiments in order to determine optimal concentrations of the drug in PLGA would be required to effectively improve the treatment and the novel delivery method.

From the statistical perspective, Bender and Lange recommended that data of exploratory studies be analyzed without multiplicity adjustment [44]. However, the lack of adjustment for multiple comparisons as well as the pilot nature of our study means that caution needs to be exercised when interpreting the results of this exploratory study. A larger study is needed to confirm these findings.

Although temporary side effects were seen, we have demonstrated that lacosamide loaded PLGA polymer sheets implanted on the surface of the cortex could affect seizure activity in GAERS by decreasing the mean duration of the SWDs events for a sustained period of up to 7 weeks. With improvements in polymer technologies and episodic release offering potentially much longer lasting release durations, intracranial polymer-based drug delivery systems may provide an effective therapeutic strategy for chronic epilepsy.

\section{Conflict of Interests}

The authors declare that they have no financial or other conflict of interests in relation to this research and its publication. 


\section{Acknowledgments}

The authors gratefully acknowledge the sponsorship of the Victorian Government through its Science Technology and Innovation Initiative administered by the Department of Industry, Innovation and Regional Development. The authors thank the Australian Research Council (ARC) for continuing financial support. Gordon Wallace thanks the ARC for his Laureate Fellowships. The authors also acknowledge use of the facilities and the assistance of Mr. Tony Romeo at the UOW Electron Microscopy Centre. The authors are grateful for access to materials synthesis and characterization equipment made available through the Australian National Fabrication Facility (ANFF).

\section{References}

[1] J. W. Sander, "The epidemiology of epilepsy revisited," Current Opinion in Neurology, vol. 16, no. 2, pp. 165-170, 2003.

[2] S. D. Shorvon, "The epidemiology and treatment of chronic and refractory epilepsy," Epilepsia, vol. 37, supplement 2, pp. S1-S3, 1996.

[3] K. Swarztrauber, S. Dewar, and J. Engel Jr., "Patient attitudes about treatments for intractable epilepsy," Epilepsy and Behavior, vol. 4, no. 1, pp. 19-25, 2003.

[4] R. S. Fisher and A. L. Velasco, "Electrical brain stimulation for epilepsy," Nature Reviews Neurology, vol. 10, no. 5, pp. 261-270, 2014.

[5] P. Kwan and M. R. Sperling, "Refractory seizures: try additional antiepileptic drugs (after two have failed) or go directly to early surgery evaluation?" Epilepsia, vol. 50, supplement 8, pp. 57-62, 2009.

[6] W. Löscher, "Mechanisms of drug resistance," Epileptic Disorders, vol. 7, supplement 1, pp. S3-S9, 2005.

[7] A. J. Halliday, S. E. Moulton, G. G. Wallace, and M. J. Cook, "Novel methods of antiepileptic drug delivery-Polymer-based implants," Advanced Drug Delivery Reviews, vol. 64, no. 10, pp. 953-964, 2012.

[8] A. J. Halliday, T. E. Campbell, T. S. Nelson, K. J. McLean, G. G. Wallace, and M. J. Cook, "Levetiracetam-loaded biodegradable polymer implants in the tetanus toxin model of temporal lobe epilepsy in rats," Journal of Clinical Neuroscience, vol. 20, no. 1, pp. 148-152, 2013.

[9] A. J. Halliday and M. J. Cook, "Polymer-based drug delivery devices for neurological disorders," CNS \& Neurological Disorders-Drug Targets, vol. 8, no. 3, pp. 205-221, 2009.

[10] A. J. Halliday, T. E. Campbell, J. M. Razal et al., "In vivo biocompatibility and in vitro characterization of poly-lactide-coglycolide structures containing levetiracetam, for the treatment of epilepsy," Journal of Biomedical Materials Research-Part A, vol. 100, no. 2, pp. 424-431, 2012.

[11] R. Groynom, E. Shoffstall, L. S. Wu, R. H. Kramer, and E. B. Lavik, "Controlled release of photoswitch drugs by degradable polymer microspheres," Journal of Drug Targeting, vol. 23, no. 7-8, pp. 710-715, 2015.

[12] M. Allahyari and E. Mohit, "Peptide/protein vaccine delivery system based on PLGA particles," Human Vaccines \& Immunotherapeutics, 2015.
[13] F. Zhang, Q. Song, X. Huang et al., "A novel high mechanical property PLGA composite matrix loaded with nanodiamondphospholipid compound for bone tissue engineering," ACS Appl Mater Interfaces, vol. 8, no. 2, pp. 1087-1097, 2016.

[14] H. Cao, M. M. Chen, Y. Liu et al., "Fish collagen-based scaffold containing PLGA microspheres for controlled growth factor delivery in skin tissue engineering," Colloids and Surfaces B: Biointerfaces, vol. 136, pp. 1098-1106, 2015.

[15] K. Yu, J. Zhao, Z. Zhang et al., "Enhanced delivery of Paclitaxel using electrostatically-conjugated Herceptin-bearing PEI/PLGA nanoparticles against HER-positive breast cancer cells," International Journal of Pharmaceutics, vol. 497, no. 1-2, pp. 78-87, 2016.

[16] C. Marescaux, M. Vergnes, and A. Depaulis, "Genetic absence epilepsy in rats from Strasbourg-a review," Journal of Neural Transmission, Supplement, vol. 35, pp. 37-69, 1992.

[17] B. K. Beyreuther, J. Freitag, C. Heers, N. Krebsfänger, U. Scharfenecker, and T. Stöhr, "Lacosamide: a review of preclinical properties," CNS Drug Reviews, vol. 13, no. 1, pp. 21-42, 2007.

[18] L. Viry, S. E. Moulton, T. Romeo et al., "Emulsion-coaxial electrospinning: designing novel architectures for sustained release of highly soluble low molecular weight drugs," Journal of Materials Chemistry, vol. 22, no. 22, pp. 11347-11353, 2012.

[19] P.-C. Lin, Y.-H. Hsieh, F.-F. Liao, and S.-H. Chen, "Determination of free and total levels of phenytoin in human plasma from patients with epilepsy by MEKC: an adequate alternative to HPLC," Electrophoresis, vol. 31, no. 9, pp. 1572-1582, 2010.

[20] S. H. Bauquier, A. Lai, J. L. Jiang, Y. Sui, and M. J. Cook, "Evaluation of an automated spike-and-wave complex detection algorithm in the EEG from a rat model of absence epilepsy," Neuroscience Bulletin, vol. 31, no. 5, pp. 601-610, 2015.

[21] T. Ishihara and T. Mizushima, "Techniques for efficient entrapment of pharmaceuticals in biodegradable solid micro/nanoparticles," Expert Opinion on Drug Delivery, vol. 7, no. 5, pp. 565-575, 2010.

[22] N. V. N. Jyothi, P. M. Prasanna, S. N. Sakarkar, K. S. Prabha, P. S. Ramaiah, and G. Y. Srawan, "Microencapsulation techniques, factors influencing encapsulation efficiency," Journal of Microencapsulation, vol. 27, no. 3, pp. 187-197, 2010.

[23] J. Kluge, M. Mazzotti, and G. Muhrer, "Solubility of Ketoprofen in colloidal PLGA," International Journal of Pharmaceutics, vol. 399, no. 1-2, pp. 163-172, 2010.

[24] S. K. Tiwari, R. Tzezana, E. Zussman, and S. S. Venkatraman, "Optimizing partition-controlled drug release from electrospun core-shell fibers," International Journal of Pharmaceutics, vol. 392, no. 1-2, pp. 209-217, 2010.

[25] S. Y. Chew, J. Wen, E. K. F. Yim, and K. W. Leong, "Sustained release of proteins from electrospun biodegradable fibers," Biomacromolecules, vol. 6, no. 4, pp. 2017-2024, 2005.

[26] W. Cui, X. Li, X. Zhu, G. Yu, S. Zhou, and J. Weng, "Investigation of drug release and matrix degradation of electrospun poly(DL-lactide) fibers with paracetanol inoculation," Biomacromolecules, vol. 7, no. 5, pp. 1623-1629, 2006.

[27] J. Zeng, L. Yang, Q. Liang et al., "Influence of the drug compatibility with polymer solution on the release kinetics of electrospun fiber formulation," Journal of Controlled Release, vol. 105, no. 1-2, pp. 43-51, 2005.

[28] Z. Xie and G. Buschle-Diller, "Electrospun poly(D,L-lactide) fibers for drug delivery: the influence of cosolvent and the mechanism of drug release," Journal of Applied Polymer Science, vol. 115, no. 1, pp. 1-8, 2010. 
[29] J.-P. A. Manning, D. A. Richards, N. Leresche, V. Crunelli, and N. G. Bowery, "Cortical-area specific block of genetically determined absence seizures by ethosuximide," Neuroscience, vol. 123, no. 1, pp. 5-9, 2004.

[30] A. A. Gurbanova, R. Aker, K. Berkman, F. Y. Onat, C. M. van Rijn, and G. van Luijtelaar, "Effect of systemic and intracortical administration of phenytoin in two genetic models of absence epilepsy," British Journal of Pharmacology, vol. 148, no. 8, pp. 1076-1082, 2006.

[31] S. H. Bauquier, A. Lai, J. L. Jiang, and M. J. Cook, "Clonic seizures induced by oral administration of enrofloxacin in GAERS," Comparative Medicine, In press.

[32] A. McRae, S. Hjorth, D. W. Mason, L. Dillon, and T. R. Tice, "Microencapsulated dopamine (DA)-induced restitution of function in 6-OHDA-denervated rat striatum in vivo: comparison between two microsphere excipients," Journal of Neural Transplantation and Plasticity, vol. 2, no. 3-4, pp. 165-173, 1991.

[33] A. McRae and A. Dahlström, "Transmitter-loaded polymeric microspheres induce regrowth of dopaminergic nerve terminals in striata of rats with 6-OH-DA induced parkinsonism," Neurochemistry International, vol. 25, no. 1, pp. 27-33, 1994.

[34] C. Gouhier, S. Chalon, M.-C. Venier-Julienne et al., "Neuroprotection of nerve growth factor-loaded microspheres on the D2 dopaminergic receptor positive-striatal neurones in quinolinic acid-lesioned rats: a quantitative autoradiographic assessment with iodobenzamide," Neuroscience Letters, vol. 288, no. 1, pp. 71-75, 2000.

[35] P. Menei, J. M. Pean, V. Nerrière-Daguin, C. Jollivet, P. Brachet, and J. P. Benoit, "Intracerebral implantation of NGF-releasing biodegradable microspheres protects striatum against excitotoxic damage," Experimental Neurology, vol. 161, no. 1, pp. 259272, 2000.

[36] J.-M. Péan, P. Menei, O. Morel, C. N. Montero-Menei, and J.P. Benoit, "Intraseptal implantation of NGF-releasing microspheres promote the survival of axotomized cholinergic neurons," Biomaterials, vol. 21, no. 20, pp. 2097-2101, 2000.

[37] B. Arica, H. S. Kaş, A. Moghdam, N. Akalan, and A. A. Hincal, "Carbidopa/levodopa-loaded biodegradable microspheres: in vivo evaluation on experimental Parkinsonism in rats," Journal of Controlled Release, vol. 102, no. 3, pp. 689-697, 2005.

[38] U. Sodemann, H. S. Møller, M. Blaabjerg, and C. P. Beier, "Successful treatment of refractory absence status epilepticus with lacosamide," Journal of Neurology, vol. 261, no. 1, pp. 20252027, 2014.

[39] C. E. Krewson, R. Dause, M. Mak, and W. M. Saltzman, "Stabilization of nerve growth factor in controlled release polymers and in tissue," Journal of Biomaterials Science, Polymer Edition, vol. 8, no. 2, pp. 103-117, 1996.

[40] C. E. Krewson, M. L. Klarman, and W. M. Saltzman, "Distribution of nerve growth factor following direct delivery to brain interstitium," Brain Research, vol. 680, no. 1-2, pp. 196-206, 1995.

[41] W. M. Saltzman, M. W. Mak, M. J. Mahoney, E. T. Duenas, and J. L. Cleland, "Intracranial delivery of recombinant nerve growth factor: release kinetics and protein distribution for three delivery systems," Pharmaceutical Research, vol. 16, no. 2, pp. 232-240, 1999.

[42] J.-C. Bensadoun, L. P. De Almeida, E. G. Fine, J. L. Tseng, N. Déglon, and P. Aebischer, "Comparative study of GDNF delivery systems for the CNS: polymer rods, encapsulated cells, and lentiviral vectors," Journal of Controlled Release, vol. 87, no. 1-3, pp. 107-115, 2003.
[43] C. Kellinghaus, "Lacosamide as treatment for partial epilepsy: mechanisms of action, pharmacology, effects, and safety," Therapeutics and Clinical Risk Management, vol. 5, no. 1, pp. 757-766, 2009.

[44] R. Bender and S. Lange, "Adjusting for multiple testing-when and how?" Journal of Clinical Epidemiology, vol. 54, no. 4, pp. 343-349, 2001. 

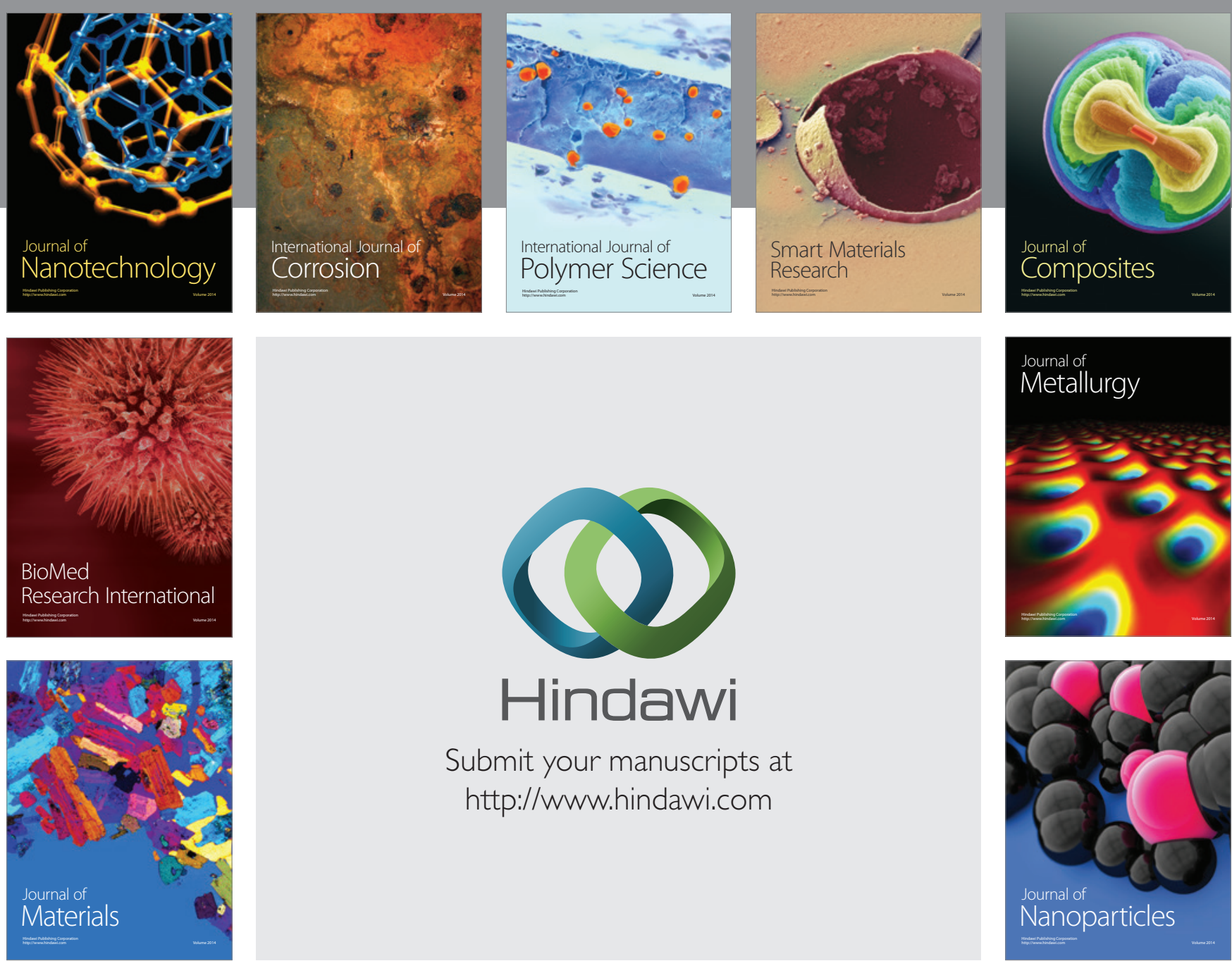

\section{Hindawi}

Submit your manuscripts at

http://www.hindawi.com

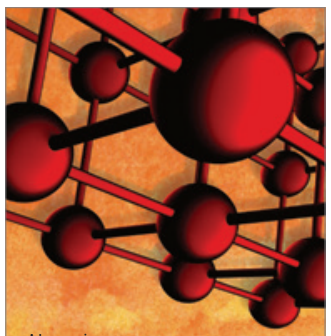

Materials Science and Engineering
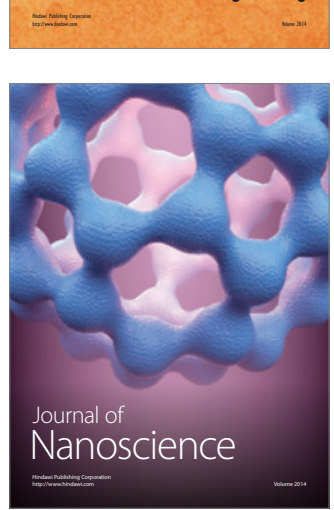
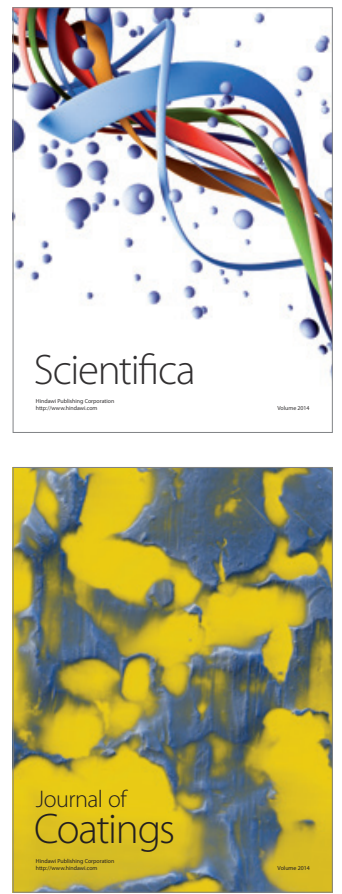
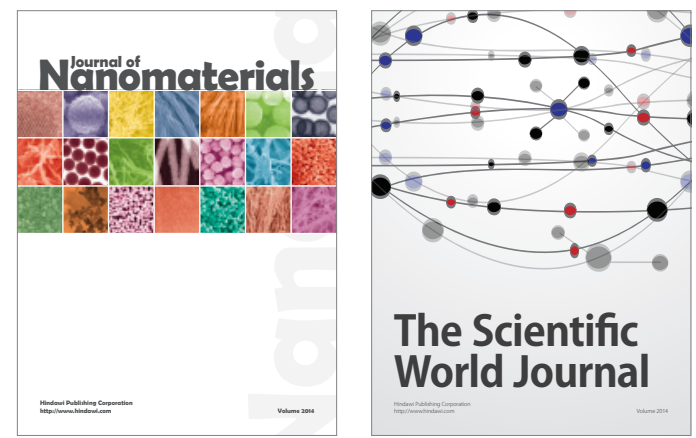

The Scientific World Journal
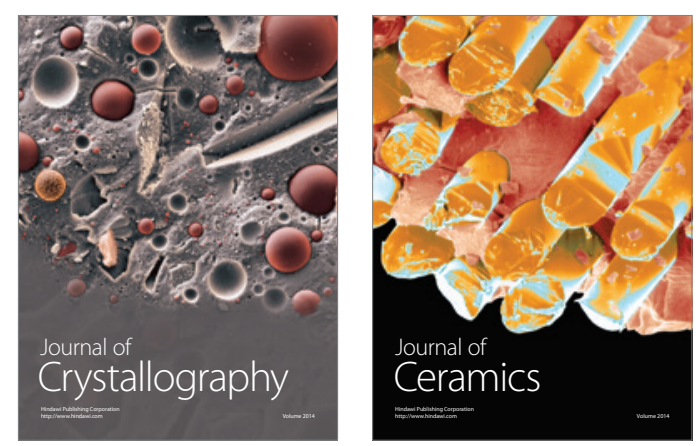
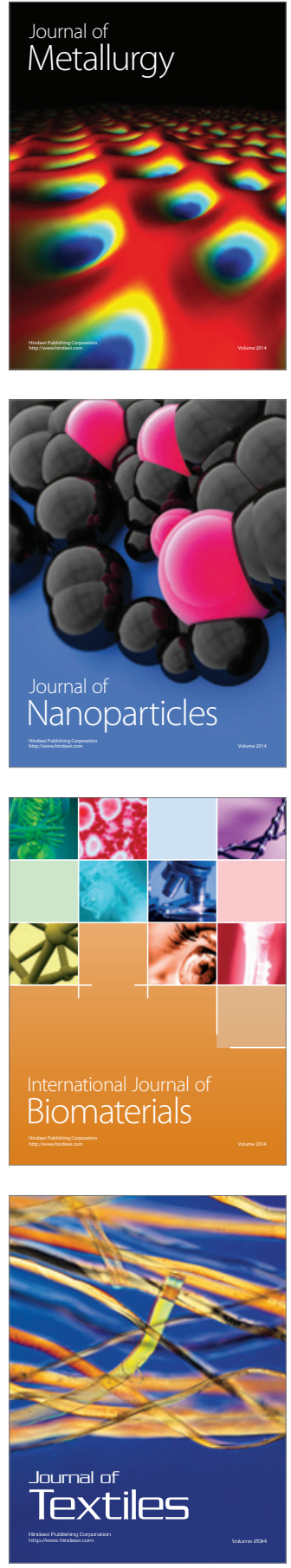